\title{
Ectoparásitos monogeneos en juveniles de Cichla monoculus (Cichlidae) de la cocha Tarapoto, río Nanay, Perú
}

\author{
Monogenean ectoparasites in juveniles of Cichla monoculus from \\ the Tarapoto lake, Nanay river, Peru
}

Humberto Arbildo Ortiz ${ }^{1,3}$, Elmer Pizango Paima ${ }^{2}$, Roger Ruiz Frias ${ }^{2}$

\section{Resumen}

\begin{abstract}
El estudio tuvo como objetivo identificar los ectoparásitos monogeneos y los índices parasitarios de juveniles de Cichla monoculus de la cocha Tarapoto, río Nanay, Perú, colectados entre julio y setiembre de 2011. Se examinaron 30 juveniles $(35.9 \pm 14.0 \mathrm{~g}$ y $14.46 \pm 1.97 \mathrm{~cm}$ ). Las aletas, fosas nasales y los arcos branquiales fueron removidos y colocados en placa petri con agua destilada, para la búsqueda de monogeneos. Los parásitos fueron fijados en glicerina amonio-picrato (GAP) para observar las estructuras esclerotizadas. Se registraron 2343 monogeneos en las branquias, colectándose 240 individuos e identificándose tres especies: Gussevia longihaptor (prevalencia $=80 \%$ intensidad media $=5.21$ parásito/pez, abundancia media $=4.17$ parásito/pez), Gussevia undulata ( revalencia $=70 \%$, intensidad media $=4.95$ parásito $/$ pez, abundancia media $=3.47$ parásito/pez) y Sciadicleithrum ergensi (prevalencia $=23.3 \%$, intensidad media $=1.57$ parásito/pez, abundancia media $=0.23$ parásito/pez). Las dos primeras especies son consideradas especies centrales, mientras que la tercera es considerada especie satélite. Así mismo, S. ergensi es reportado por primera vez en el Perú y en Cichla monoculus.
\end{abstract}

Palabras clave: Cichla monoculus, monogeneos, cocha Tarapoto, Nanay, Perú

\section{AbSTRACT}

The aim of this study was to identify the monogenean ectoparasites and the parasitic indices of juveniles of Cichla monoculus from the Tarapoto lake, Nanay river, Peru, between July and September 2011. Thirty juveniles were examined (35.9 $\pm 14.0 \mathrm{~g}$ and 14.46 $\pm 1.97 \mathrm{~cm}$ ). The fins, nostrils and branchial arches were removed and placed in a petri dish

\footnotetext{
${ }^{1}$ Programa de Maestría en Ciencias con mención en Acuicultura, Universidad Nacional de la Amazonia Peruana, Iquitos, Perú

${ }^{2}$ Departamento Académico de Hidrobiología, Facultad de Ciencias Biológicas, Universidad Nacional de la Amazonia Peruana, Iquitos, Perú

${ }^{3}$ E-mail:doc.arbil@gmail.com
}

Recibido: 11 de enero de 2019

Aceptado para publicación: 29 de enero de 2020

Publicado: 31 de marzo de 2020 
with distilled water for the search of monogeneans. The parasites were fixed in glycerin ammonium-picrate (GAP) to observe the sclerotized structures. There were 2343 monogeneans in the gills and 240 individuals were collected for identification. Three species were identified: Gussevia longihaptor (prevalence $=80 \%$, mean intensity $=5.21$ parasite/fish, mean abundance $=4.17$ parasite/fish), Gussevia undulata (prevalence $=$ $70 \%$, mean intensity $=4.95$ parasite $/$ fish, mean abundance $=3.47$ parasite $/$ fish $)$ and Sciadicleithrum ergensi (prevalence $=23.3 \%$, mean intensity $=1.57$ parasite $/$ fish, mean abundance $=0.23$ parasite/fish). The first two species are considered central species, while the third is considered a satellite species. Likewise, S. ergensi is reported for the first time in Peru and in Cichla monoculus.

Key words: Cichla monoculus, monogeneans, cocha Tarapoto, Nanay, Perú

\section{INTRODUCCIÓN}

La región amazónica tiene alrededor de $6112000 \mathrm{~km}^{2}$, representando aproximadamente a la tercera parte de la superficie de América del Sur (Granado, 2000). La ictiofauna de la cuenca amazónica contiene entre 2500 y 3000 especies (Junk y Soares, 2001), habiendo más de 800 especies en la amazonia peruana (Ortega et al., 2012).

Cichla monoculus «tucunaré» es una especie que habita en ambientes lénticos y alcanza mayor talla dentro de la familia Cichlidae en el Neotrópico, siendo la talla mínima de madurez sexual entre 23 y $27 \mathrm{~cm}$, y tiene un hábitat alimenticio carnívoro con tendencia a piscívoro (García et al., 2018). En la amazonia peruana, esta especie es capturada de los ambientes naturales por la buena calidad de carne que presenta y es comercializada en los mercados en el estado de fresco y salado (Riofrío et al., 2000).

En el ambiente acuático se encuentra una amplia variedad de parámetros fisicoquímicos y biológicos que influyen sobre el crecimiento y reproducción de los peces. En los casos en que las condiciones se tornan desfavorables para los peces, se produce un descenso en sus defensas naturales y los patógenos, incluyendo los parásitos, invaden a los hospederos (Centeno et al.,
2004). Dentro del grupo de parásitos que causan lesiones y mortandad en los peces están los monogeneos, tanto especies marinas como de agua continental (Pavanelli et al., 1999; Flores J y Flores R, 2003). Estos parásitos tienen un ciclo de vida directo y el daño producido al hospedero dependerá del número de individuos que se localicen en las branquias $\mathrm{u}$ otras partes del cuerpo.

En Perú son pocos los estudios relacionados a los monogeneos presentes en los peces cíclidos procedentes de ambientes naturales y artificiales, especialmente aquellos de la amazonia peruana. Entre las especies de ambiente natural que se han investigado se encuentran Heros severus, Astronotus ocellatus, Cichlasoma amazonarum, Cichla monoculus y Satanoperca jurupari (Mendoza-Franco et al., 2010), mientras que del ambiente controlados se encuentran Cichla monoculus y Chaetobranchus semifasciatus (Mathews et al., 2012, 2013).

Por otro lado, el conocimiento de las especies de parásitos de peces de ambiente natural y de cultivo en la región Loreto es muy pobre, con consecuencias negativas para el desarrollo de la piscicultura (Scholz, 2008). Por lo expuesto, y siendo Cichla monoculus un pez ornamental y de consumo, con interés en el cultivo en ambientes controlados, el presente trabajo tuvo como finalidad identificar los monogeneos, determinar los índices para- 
sitarios y la relación parásito-hospedero en juveniles de Cichla monoculus procedentes de ambientes naturales.

\section{Materiales y Métodos}

\section{Localización del Estudio}

El trabajo se realizó con peces obtenidos de la cocha Tarapoto del río Nanay, específicamente en las áreas aledañas a la comunidad de Santa Clara $\left(3^{\circ} 47^{\prime} 71^{\prime \prime S}\right.$, $73^{\circ} 21^{\prime} 13.2^{\prime \prime}$ ), al sureste de la ciudad de Iquitos, departamento de Loreto, Perú.

Un total de 30 especímenes de Cichla monoculus con peso promedio de $35.9 \pm 14.0$ $\mathrm{g}$ y longitud total promedio de $14.46 \pm 1.97$ $\mathrm{cm}$, clasificados como juveniles por el tamaño y la observación de los órganos reproductivos (Riofrío et al., 2000) fueron capturados quincenalmente, desde julio a septiembre de 2011 (10 especímenes por mes). La captura se realizó en horas de la mañana con una red anchovetera de $20 \mathrm{~m}$ de largo, 2 $m$ de ancho y $2 "$ de abertura. Los peces fueron acondicionados en bolsas plásticas con agua y aire, y trasladados al Laboratorio de Investigación de la Facultad de Ciencias Biológicas de la Universidad Nacional de la Amazonia Peruana (UNAP), Iquitos, donde se realizaron las necropsias y análisis correspondientes

\section{Necropsia de los Peces}

Para la necropsia de los peces se siguió la metodología indicada por Eiras et al. (2006). Los peces fueron sacrificados mediante una perforación en la región cefálica con la ayuda de un estilete, luego se registró la longitud total $(\mathrm{cm})$ y el peso $(\mathrm{g})$, utilizando un ictiómetro de madera de $50 \mathrm{~cm}$ y una balanza digital (OHAUS) de 600 g capacidad y $0.01 \mathrm{~g}$ de sensibilidad.

Se observó macroscópicamente la superficie externa del pez, explorando las partes laterales, así como las aletas, buscando la presencia de ectoparásitos, con ayuda de un estereoscopio. Posteriormente, con una espátula se realizó un raspado de la piel, desde la parte anterior hasta la posterior y se cortaron los extremos de las aletas (dorsal, pectoral, anal y caudal) para luego ser observadas al microscopio. Asimismo, se seccionó el opérculo derecho del pez, para extraer las branquias, colocándolas en placas petri con agua destilada, las cuales fueron examinadas con la ayuda de microscopio compuesto Nikon E2000 Eclipse.

\section{Monogeneos}

Se contaron todos los monogeneos a nivel de clase presentes en los 30 juveniles de Cichla monoculus. El lugar de fijación de los parásitos se determinó mediante la observación directa de las estructuras del pez en el momento de la colecta. Luego se colectaron ocho monogeneos de cada pez (total: 240 especímenes), para identificar a nivel de especie. Dado el alto número de monogeneos colectados se trabajó con la Muestra Sistemática, que consiste en seleccionar aleatoriamente los individuos dentro de la muestra (Husch et al., 1993; Péllico Neto y Brena, 1997).

Los parásitos fueron fijados con glicerina amonio-picrato (GAP) de acuerdo con Malmberg (1957), para el estudio de las estructuras esclerotizadas: anclas, barras dorsales y ventrales, ganchitos marginales, complejo copulador y la esclerita vaginal presente.

Para la identificación de los parásitos se utilizaron los trabajos de Mendoza-Franco et al. (2010) y la «Clave para monogeneos neotropicales de agua dulce» descrita por Thatcher (2006). Los especímenes representativos fueron depositados en la colección helmintológica de la Facultad de Ciencias Biológicas, UNAP.

\section{Análisis de los Datos}

Para el análisis cuantitativo de los parásitos encontrados se utilizaron los índices parasitarios prevalencia, intensidad media y 

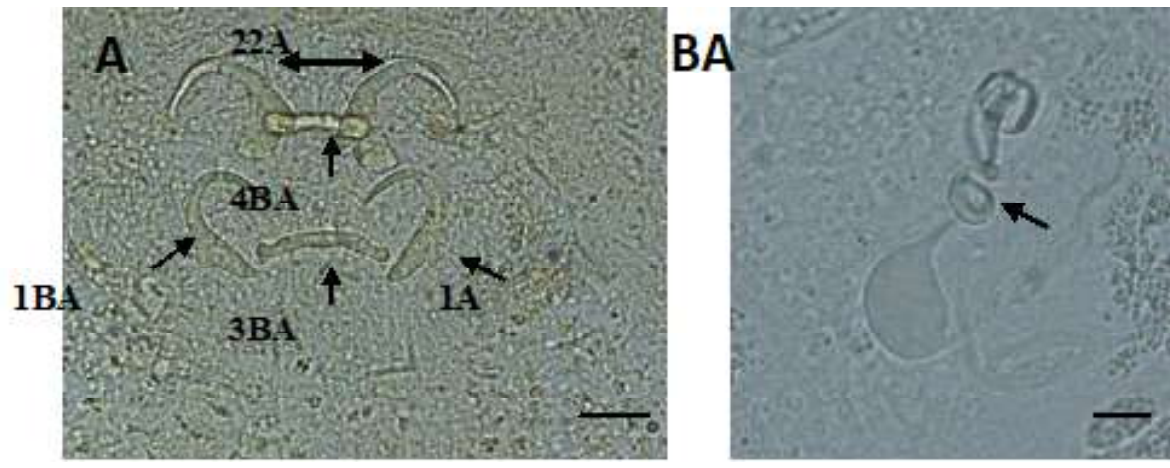

Figura 1. Estructuras esclerotizadas de Gussevia longihaptor (A): 1-ancla dorsal, 2-ancla ventral, 3-barra dorsal, 4-barra ventral y complejo copulatorio; (B): (Técnica de Malmberg). Escala de la barra $=10 \mu \mathrm{m}$
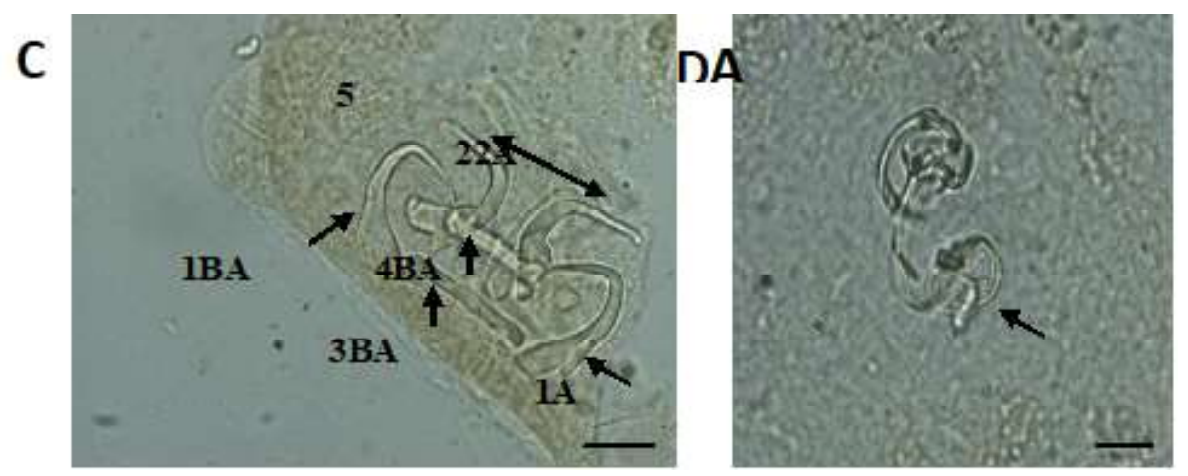

Figura 2. Estructuras esclerotizadas de Gussevia undulata (C): 1-ancla dorsal, 2-ancla ventral, 3-barra dorsal, 4-barra ventral, 5-ganchitos y complejo copulatorio; (D): (Técnica de Malmberg). Escala de la barra $=10 \mu \mathrm{m}$

abundancia media, según Bush et al. (1997). Los componentes de la comunidad parasitaria fueron clasificados de acuerdo con Bush y Holmes (1986) en especies centrales (presentes en más de dos tercios de los hospederos), especies secundarias (presentes en uno a dos tercios del hospedero) y satélites (presentes en menos de un tercio de los hospederos). Asimismo, para determinar la relación del peso y longitud de los hospederos con la abundancia de los parásitos se utilizó el coeficiente de correlación de Pearson $(r)$ a una significancia de $\mathrm{p}<0.05$, mediante el programa estadístico Sigma Plot 11.

\section{Resultados}

Se encontraron tres especies de monogeneos en las branquias, Gussevia longihaptor (Figura 1), Gussevia undulata (Figura 2), Sciadicleithrum ergensi (Figura 3). No se registró la presencia de monogeneos en la piel, aletas y fosas nasales. Todos los peces estuvieron parasitados por monogeneos. Se registraron 2343 monogeneos, la intensidad media fue de 156.2 monogeneos/pez parasitado y la abundancia fue de 156.2 monogeneos/pez. 

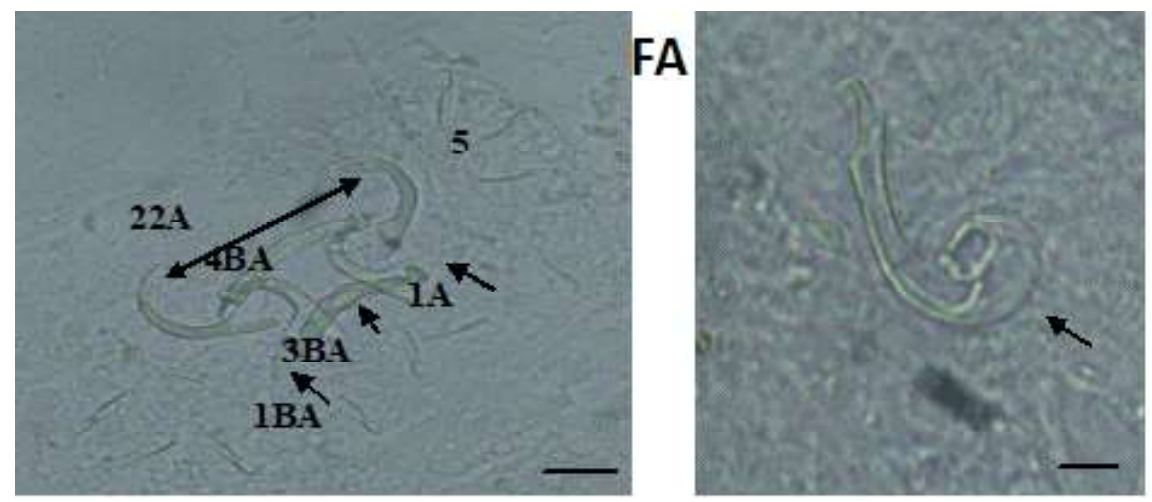

Figura 3. Estructuras esclerotizadas de Sciadicleithrum ergensi (E): 1-ancla dorsal, 2-ancla ventral, 3-barra dorsal, 4-barra ventral, 5-ganchitos y complejo copulatorio; (F): (Técnica de Malmberg). Escala de la barra $=10 \mu \mathrm{m}$
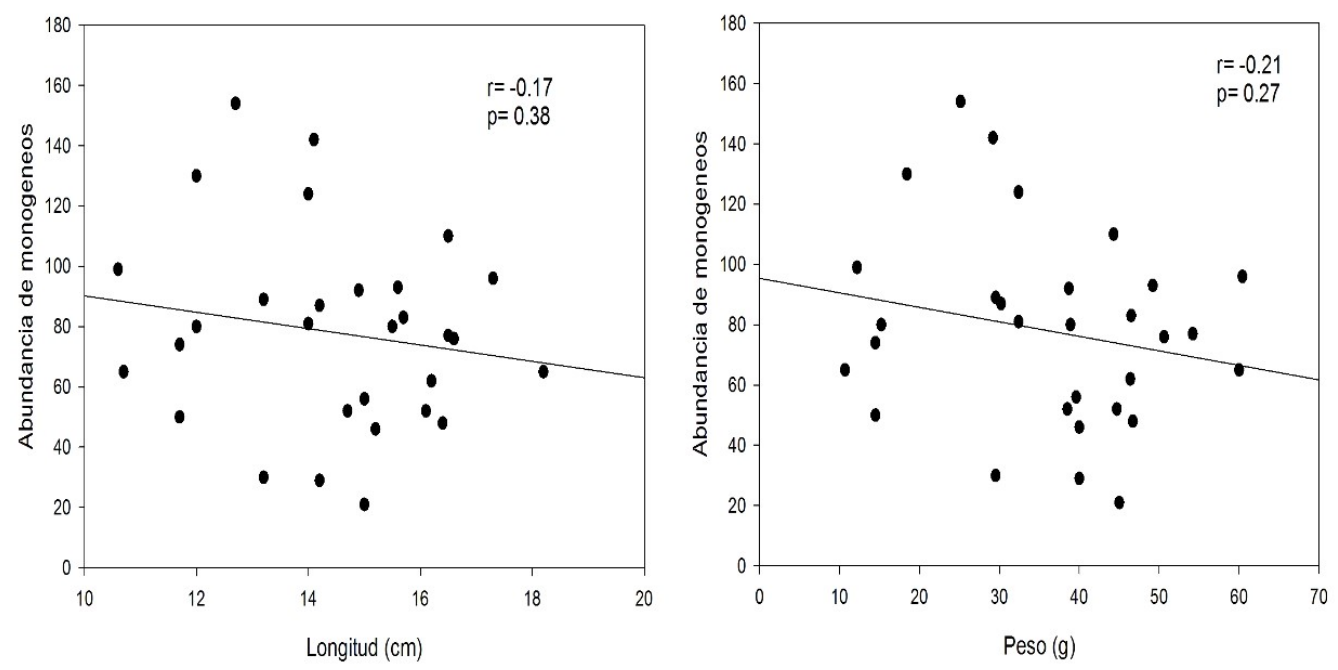

Figura 4. Coeficiente de correlación de Pearson entre la abundancia de monogeneos en juveniles de Cichla monoculus y la longitud total (izquierdo), y el peso (derecho)

En el Cuadro 1 se muestran los resultados de los índices parasitarios de los 240 monogeneos colectados y fijados en láminas permanentes. La especie más prevalente fue G. longihaptor, presente en el $80 \%$ de los peces. Las dos especies de Gussevia son consideradas especies centrales y $S$. ergensi como especie secundaria. Por otro lado, no se registró correlación significativa entre la abundancia de monogeneos con la longitud total $(\mathrm{r}=-017 ; \mathrm{p}=0.38)$ y el peso $(\mathrm{r}=-0.21$; $\mathrm{p}=0.27$ ) (Figura 4 ).

\section{Discusión}

En el estudio filogenético de las especies del género Sciadicleithrum, realizado por Mendoza-Franco y Vidal-Martínez (2005) se concluye que 15 especies de este género infectan a 13 especies de cíclidos de Centro y Sur América, mientras que solo cuatro especies de Sciadicleithrum infectan a 14 especies de cíclidos en el sureste de México. Por otro lado, 17 especies del género Gussevia 
Cuadro 1. Índices parasitarios, estatus comunitario y lugar de fijación de tres especies de monogeneos encontrados en juveniles de Cichla monoculus provenientes de la Cocha Tarapoto en el río Nanay. Loreto, Perú

\begin{tabular}{lccc}
\hline Índices evaluados $^{1}$ & $\begin{array}{c}\text { Gussevia } \\
\text { longihaptor }\end{array}$ & $\begin{array}{c}\text { Gussevia } \\
\text { undulata }\end{array}$ & $\begin{array}{c}\text { Sciadicleithrum } \\
\text { ergensi }\end{array}$ \\
\hline Peces examinados (n) & 30 & 30 & 30 \\
Peces infectados (n) & 24 & 21 & 7 \\
Parásitos colectados (n) & 125 & 104 & 11 \\
Prevalencia (\%) & 80 & 70 & 23.2 \\
Intensidad media & 5.21 & 4.95 & 1.57 \\
Abundancia media & 4.17 & 3.47 & 0.27 \\
Status comunitario & Central & Central & Secundaria \\
Lugar de fijación & Branquias & Branquias & Branquias \\
\hline
\end{tabular}

${ }^{1}$ Datos de 240 monogeneos seleccionados de una muestra total de 2343 monogeneos encontrados en $\Xi$ juveniles de Cichla monoculus

en América del Sur han sido descritos en siete especies de cíclidos (Thatcher, 2006).

Especies de los géneros Gussevia y Sciadicleithrum han sido reportadas en cinco especies de cíclidos de importancia económica en la amazonia peruana; entre ellas se encuentran Gussevia alioides (Heros severus), Gussevia asota (Astronotus ocellatus), Gussevia disparoides ( $H$. severus y Cichlasoma amazonarum), Gussevia longihaptor, Gussevia undulata, Tucunarella cichla (C. monoculus), Sciadicleithrum satanopercae (Satanoperca jurupari) y Sciadicleithrum variabilum (C. amazonarum) (Mendoza-Franco et al., 2010; Mathews et al., 2012).

En la literatura revisada para Cichla monoculus se han reportado 11 monogeneos, tres a nivel de género, Notozothecium $\mathrm{sp}$ (Müller et al., 2008), Gussevia sp y Sciadicleithrum sp (Santana et al., 2017) y ocho a nivel de especies, Gussevia arilla, $G$. longihaptor, G. undulata, G. tucunarense, Sciadicleithrum ergensi, S. umbiculum, S. uncinatum y Tucunarrella cichla (MendozaFranco et al., 2010; Mathews et al., 2013a; Santana et al., 2017). En este estudio se en- contraron tres parásitos monogeneos branquiales, G. longihaptor, G. undulata y $S$. ergensi. Estas especies presentan las características descritas originalmente por Kristky et al. (1989). El bajo número de especies registradas en este trabajo pude atribuirse al número de monogeneos seleccionados para la identificación, dado que en este estudio se utilizaron ocho monogeneos por cada espécimen, mientras que los demás autores identificaron todos los especímenes presentes en los hospederos. Por otro lado, estas tres especies han sido registradas en Brasil y México en Cichla ocellaris, Cichla kelberi y Cichlasoma urophthalmus (Kristky et al., 1986, 1989; Pérez-Ponce et al., 1996; Abdallah, 2009; Takemoto et al., 2009; Yamada et al., 2011).

El monogeneo S. ergensi, encontrado en el presente trabajo, representa un nuevo huésped para $C$. monoculus y, por consiguiente, se estaría extendiendo su distribución geográfica. Asimismo, para el Perú es el primer registro de la presencia de este parásito y en juveniles de $C$. monoculus. Los parásitos de los géneros Gussevia y Sciadicleithrum son específicos para cíclidos, 
lo que se confirma con el resultado de este estudio. Esta especificidad se debe a que los monogeneos son un grupo de parásitos que han co-evolucionado junto a sus huéspedes (Simková et al., 2006).

En general, los parásitos monogeneos en los peces se pueden encontrar en las branquias, cuerpo, aletas y fosas nasales (Thatcher, 2006). No obstante, en este estudio solo estuvieron presentes en las branquias coincidiendo con los resultados de MendozaFranco et al. (2010), Mathews et al. (2013a,b) y Santana et al. (2017. Los monogeneos registrados pertenecen a la familia Dactylogyridae, las cuales tienen preferencia por las branquias de los hospederos, a diferencia de la familia Gyrodactylidae, quienes prefieren la piel y las aletas (Thatcher, 2006). Los monogeneos pueden producir erupciones focales, aneurisma, hemorragias, reacciones inflamatorias, hiperplasia de células epiteliales y excesiva producción de moco, reduciendo la capacidad respiratoria (Eslava e Iregui, 2000). El daño producido por los monogeneos en los peces está relacionado con la especie, lugar de la infestación, número de individuos encontrados en las branquias u otras partes del cuerpo, así como por el tipo de alimentación, ya que la mayoría se alimenta de mucus y células epiteliales, aunque algunos se alimentan de sangre (Pavanelli et al., 1999; Thatcher, 2006).

Los valores similares de abundancia media e intensidad media encontrada en el presente estudio (156.2 monogeneos/pez) a nivel de clase se debe a que el número de peces analizados fueron iguales a los peces infectados. El registro de valores elevados de prevalencia es común de ser observado en peces procedentes de cultivo en la amazonia peruana (Mathews et al., 2013a,b). En temperaturas ambientales elevadas, el ciclo biológico de estos parásitos puede realizarse en menos de un día, o durar de uno a cinco días (Flores J y Flores R, 1993, 2003; Flores-Crespo et al., 1995). Las especies de monogeneos tienen una producción anual de infección bien definida, con aumento del número de parásitos en verano y una reducción en los meses de invierno (Eiras, 1994).

G. longihaptor y G. undulata fueron las especies de monogeneos que presentaron valores elevados de índice parasitarios en comparación con S. ergensi (Cuadro 1). En G. undulata se registró una prevalencia de $80 \%$ e intensidad de 4.5 por pez infectado, mientras que Mathews et al. (2012), en la misma especie, pero en diferente estadio y ambiente (estanque de tierra), registra valores elevados de prevalencia (100\%) e intensidad (168.5 monogeneo/pez infectado), diferencia que pudo haber estado influenciada por las condiciones ambientales del lugar y época de colecta, así como por la cantidad de especímenes de G. undulata colectados. En este estudio se colectaron 104 especímenes, mientras que el otro autor colectó 9864 especímenes.

Referente a $S$. ergensi, los valores del Cuadro 1 son bajos al ser comparados con los registrados por Santana et al. (2017) en peces procedentes de seis lagos de la amazonia central en Brasil, quienes registraron una intensidad de 68 especímenes, prevalencia de $50 \%$, intensidad media de 3.58 por pez infestado y coincidiendo con el status comunitario de secundario.

Los peces bajo cultivo en ambientes controlados son propensos a infestaciones elevadas de ectoparásitos, producto del desequilibrio hospedero-parásito-ambiente e influenciado por la acumulación de la materia orgánica, producto de una elevada densidad de siembra, así como de alimentación y medidas profilácticas deficientes (Dias et al., 2015). Por otro lado, en la cocha Tarapoto, los lagos y los estanques de cultivo semi-intensivos, donde fueron colectados los hospederos en este estudio y por los autores mencionados, son ambientes lénticos; ambiente que favorece la transmisión de los parásitos de ciclo de vida directo como los monogeneos (Flores et al., 2003; Pavanelli et al., 2004; Azevedo et al., 2007). 
La prevalencia está influenciada por factores externos tales como el encuentro entre hospedadores y etapas infectivas de los parásitos y la oferta de especies hospedadoras disponibles, entre otros, mientras que la intensidad de infestación está determinada por procesos inherentes al pezy al parásito, tales como el tamaño relativo del sitio de infestación y la talla del parásito, que constituyen factores denso-dependientes determinantes de un rango de tolerancia (Poulin, 2006).

En este trabajo no hubo una correlación positiva significativa entre la longitud total y el peso con la abundancia de los monogeneos, siendo diferente a lo registrado por Santana et al. (2017), quienes colectaron 38 ejemplares de C. monoculus. Así mismo, Yamada et al. (2011) registraron una correlación positiva significativa entre longitud de Cichla piquiti y Cichla kelberi con la abundancia de $S$. ergensi.

\section{Conclusiones}

- Se registraron tres parásitos monogeneos Gussevia undulata, G. longihaptor y Sciadicleithrum ergensi, presentes en las branquias de juveniles Cichla monoculus, siendo $S$. ergensi un nuevo registro para Perú.

- Los parásitos monogeneos Gussevia undulata y G. longihaptor registraron valores elevados de prevalencia, siendo considerados especies centrales.

- No hubo correlación significativa entre el peso y la longitud total con la abundancia de los monogeneos.

\section{Agradecimiento}

Al Dr. Thomas Scholz y al Dr. Carlos Mendoza Palmero, investigadores del Instituto de Parasitología, Academia de Ciencias de la República Checa, por la contribución en la confirmación de las especies de parásitos encontradas en el presente trabajo y el aporte de literaturas específicas.

\section{Literatura Citada}

1. Abdallah VD. 2009. Biodiversidade dos monogenéticos (Platyhelminthes: Monogenea) parásitos de peixes do rio Guandú, Estado do Rio de Janeiro, Brasil. Tesis Doctoral. Brasil: Universidade Federal Rural do Rio de Janeiro. 87 p.

2. Azevedo RK, Abdallah VD, Luque JL. 2007. Ecología da comunidade de metazoários parásitos do Apaiarí Astronotus ocellatus (Cope, 1872) (Perciformes: Cichlidae) do rio Guandú, Estado de Rio de Janeiro, Brasil. Rev Bras Parasitol V 16: 15-20.

3. Bush AO, Holmes JC. 1986. Intestinal helminths of lesser scaup ducks: an interactive community. Can J Zool 54: 142-154. doi: 10.1139/z86-023

4. Bush AO, Lofferty KD, Lozt JM, Shostak AW. 1997. Parasitology meets ecology on its own terms: Margolis et al. revisited. J Parasitol 83: 575-583. doi: $10.2307 / 3284227$

5. Centeno L, Silva-Acuña A, Silva-Acuña R, Pérez JL. 2004. Fauna ectoparasitaria asociada a Colossoma macropomum y al hibrido de C. macropomum x P. brachypomus, en cultivos en estado Delta Amacuro Venezuela. Bioagro 16: 121-126.

6. Dias MKR, Neves LR, Marinho R, Pinheiro DA, Tavares-Dias M. 2015. Parasitismo em tambatinga (Colossoma macropomum x Piaractus brachypomus, Characidae) cultivados na Amazônia, Brasil. Acta Amazon 45: 231-238. doi: 10.1590/1809-4392201400974

7. Eiras JC. 1994. Elementos de ictioparasitologia. Porto: Fundação Eng. Antônio de Almeida. 339 p.

8. Eiras JC, Takemoto RM, Pavanelli GC. 2006. Método de estudo y técnicas laboratoriais em parasitología de peixes. Brasil: Universidade Estadual de Maringá. 199 p.

9. Eslava PR, Iregui CA. 2000. Estudios sobre las enfermedades branquiales en la cachama blanca (Piaractus brachypomus). Orinoquia 4: 123-151. 
10. Flores J, Flores R. 1993. Principales trematodos y cestodos de importancia económica en acuicultura. En: Tópicos de parasitología animal cestodos y trematodos. México: Univ. Nacional Autónoma del Estado de Morelos II. p 13-25.

11. Flores J, Flores R. 2003. Monogeneos, parásitos de peces en México: estudio recapitulativo. Rev Mex Cienc Pecu 41: 175-192.

12. Flores-Crespo J, Flores-Crespo $R$, Ibarra-Velarde F, Vera-Montenegro $Y$, Vásquez-Peláez C. 1995. Evaluation of chemotherapeutic agents against cichlidogyriasis in tilapia (Oreochromis hornorum) in Mexico. Rev Latinoam Microbiol 37: 179-187.

13. García-Dávila C, Sánchez H, Flores M, Mejia J, Angulo C, Castro-Ruiz D, Estivals G, et al. 2018. Peces de consumo de la Amazonía peruana. Iquitos, Perú: Instituto de Investigaciones de la Amazonía Peruana (IIAP). 218 p.

14. Granado LC. 2000. Ecología de comunidades: el paradigma de los peces de agua dulce. Sevilla, España: Univ. de Sevilla. $284 \mathrm{p}$.

15. Husch B, Miller CI, Beers TW. 1993. Forest mensuration. $3^{\text {rd }}$ ed. Malabar: Krieger Publ. 402 p.

16. Junk WJ, Soares MGM. 2001. Freshwater fish habitats in Amazonia: state of knowledge, management, and protection. Aquat Ecosyst Health 4: 437451. doi: 10.1080/146349801317276107

17. Malmberg G. 1957. Om forekomsten av Gyrodactylus pa svenska fiskar. Skr sod Sver Fisk For Arsskr 19: 19-76.

18. Mathews PD, Mathews DJP, Ismiño OR. 2012. Massive infestation by Gussevia undulata (Platyhelminthes: Monogenea: Dactylogyridae) in fingerlings of Cichla monoculus cultured in the Peruvian Amazon. Neotrop Helminthol 6: 231-237.

19. Mathews PD, Mertins O, Mathews JP, Orbe RI. 2013a. Massive parasitism by Gussevia tucunarense (Platyhelminthes: Monogenea: Dactylogyridae) in finger- lings of bujurqui-tucunare cultured in the Peruvian Amazon. Acta Parasitol 58: 223225. doi: 10.2478/s11686-013-0129-7

20. Mathews PD, Mathews JP, Ismiño RO. 2013b. Parasitic infections in juveniles of Prochilodus nigricans farm in the Peruvian Amazon. Bull Eur Assn Fish P 33: 28-32.

21. Mendoza-Franco EF, Vidal-Martínez VM. 2005. Phylogeny of species of Sciadicleithrum (Monogenoidea: Ancyrocephalinae), and their historical biogeography in the Neotropics. J Parasitol 91: 253-259. doi: 10.1645/GE-3389

22. Mendoza-Franco, Scholz, T, Rozkosná P. 2010. Tucunarella n. Gen. and other dactylogyrids (Monogenoidea) from cichlid fish (Perciformes) from Peruvian Amazonia. J Parasitol 96: 491-498. doi: 10.1645/GE-2213.1

23. Müller MI, Madi RR, Ueta MT. 2008. Primeiro registro de o correncia de cestódeos da familia Bothriocephalidae Blanchard, 1849 (Pseudophyllidea), parasitando Cichla monoculus (Cichlidae) nas lago as da Fazenda Rio das Pedras, Campinas (SP). Bioikos 22: 45-49.

24. Ortega H, Hidalgo M, Corea E, Espino J, Chocano L, Trevejo G. Meza $V$, et al. 2011. Lista anotada de los peces de aguas continentales del Perú: estado actual del conocimiento, distribución, usos y aspectos de conservación. Lima, Perú: Ministerio del Ambiente, Museo de Historia Natural - UNMSM. 48 p.

25. Pavanelli GC, Eiras JC, Takemoto RM. 1999. Doencas de peixes. Profilaxia, diagnóstico e tratamiento. Maringa, Brasil: Univ. Estadual de Maringá. 264 p.

26. Pavanelli GC, Machado MH, Takemoto, RM, Guidelli, GM, Lizama MAP. 2004. Helminth fauna of the fishes: diversity and ecological aspects. In: Thomaz SM, Agostinho AA, Hahn NS (eds). The upper Paraná river and its floodplain: physical aspects, ecology and conservation. Leiden: Backhuys. p 309-329.

27. Pellico Neto S, Brena DA. 1997. Inventário florestal. Curitiba: EMBRAPA. 316 p. 
28. Pérez-Ponce GDL, García-Prieto L, Osorio-Sarabia D, León-Régagnon $V$. 1996. Helmintos parásitos de peces de agua continentales de México. México: Univ. Nacional Autónoma de México. $100 \mathrm{p}$.

29. Poulin R. 2006. Variation in infection parameters among populations within parasite species: intrinsic properties versus local factors. Int J Parasitol 36: 877 885. doi: 10.1016/j.ijpara.2006.02.021

30. Riofrío JC, Zaldívar JE, Villanueva CA, Velarde DA. 2000. Biología pesquera, extracción y uso potencial de «tucunare» (Cichla monoculus, Pisces: Cichlidae) en Ucayali. Rev Peru Biol 7: 142-150.

31. Santana H, Murrieta G, Malta J. 2017. Especies de monogenoidea parásitos de las Branquias de Cichla monoculus (Spix \& Agassiz, 1831) (Perciformes: Cichlidae) de lagos de varzea de la amazonia de Brasil. Neotrop Helminthol $11:$ 129-138.

32. Šimková OV. 2006. Specificity and specialization of congeneric monogeneans parasitizing cyprinid fish. Evolution 60: 1023-1037.

33. Scholz T. 2008. Research on fish parasites of the upper Amazon River basin: current state and perspective. En: XIV Jornada de Acuicultura. Colombia.

34. Takemoto RM, Pavanelli GC, Lizama MAP, Lacerda ACF, Yamada FH, et al. 2009. Diversity of parasites of fish from the Upper Paraná River floodplain, Brazil. Braz J Biol 69: 691-705.

35. Thatcher VE. 2006. Amazon fish parasites. $2^{\text {nd }}$ ed. Moscow: Pensoft. 507 p.

36. Yamada FH, Santos LN, Takemoto RM. 2011. Gill ectoparasites assemblages of two nonnative Cichla populations (Perciformes, Cichlidae) in Brazilian reservoirs. J Helminthol 85: 185-191. doi: 10.1017/S0022149X10000441 